\title{
Aspectos preliminares del manejo reproductivo en cautiverio de la doncella (Ageneiosus pardalis Lütken, 1874)
}

\section{Preliminary aspects of the reproductive handling in captivity of doncella (Ageneiosus pardalis Lütken, 1874)}

\author{
Pedro Contreras C, ${ }^{1 *}$ Biol Mar, Beatriz Zapata $\mathrm{B}^{2}{ }^{2}$ Esp, Rafael Rosado $\mathrm{P}^{3}$ M.Sc.
}

${ }^{1}$ INCODER-ISAGEN-Corporación Programa para la paz del Magdalena Centro, Avenida El Dorado - CAN, Bogotá. 'Universidad de los Llanos, Instituto de Acuicultura de los Llanos, km 12 vía Puerto López, Villavicencio, Colombia. ${ }^{3}$ Truchas de la Sierra, AA 85194, Bogotá. *Correspondencia: rrosadop@ gmail.com

Recibido: Abril de 2011; Aceptado: Febrero de 2012.

\begin{abstract}
RESUMEN
Objetivo. Evaluar procedimientos de manejo en cautiverio de ejemplares maduros de doncella Ageneiosus pardalis, con fines de reproducción y presentar una descripción preliminar del desarrollo embrionario y larvario para la especie. Materiales y métodos. Sobre ejemplares mantenidos en cautiverio se adelantaron dos ensayos de reproducción, así: a) inducción con extracto de hipófisis de carpa EHC para la espermiación ( $4 \mathrm{mg} / \mathrm{kg}$; una dosis intraperitoneal) y para la ovulación ( 0.5 y $5 \mathrm{mg} /$ kg; intraperitoneales, 0 y 12 horas); b) inducción únicamente sobre hembras mantenidas en piletas, sin machos presentes, recolección y seguimiento de ovas. Resultados. Se comprobó la ovulación y la espermiación, pero no se avanzó en el desarrollo embrionario cuando se mezclaron los gametos y fueron incubados. Los ensayos con hembras inducidas, sin machos presentes, confirman el almacenamiento de esperma en los ovarios y los huevos obtenidos mostraron desarrollo embrionario completo, lográndose eclosión de larvas y el mantenimiento de alevinos. Conclusiones. El protocolo convencional de inducción fue efectivo en hembras y machos, pero la mezcla de los gametos no resultó ser un procedimiento viable para la producción de semilla en la especie. Se comprueba el almacenamiento de esperma en las hembras y la emisión espontánea de huevos fertilizados al medio cuando estas son sometidas a inducción. Tanto la forma y el tiempo de almacenamiento de esperma como los mecanismos fisiológicos que ocurren en la fecundación se desconocen.
\end{abstract}

Palabras clave: Auchenipteridae, reproducción, siluriformes (Fuente: AIMS). 


\section{ABSTRACT}

Objective. To evaluate handling procedures in captivity of mature specimens, with reproduction purposes, of doncella Ageneiosus pardalis, and to present the first advance in the description of embryonic and larval development for the species. Materials and methods. Several reproduction tests were carried out in specimens maintained in captivity, as follows: a) Spermiation and ovulation were induced with Carp pituitary extract CPE ( $4 \mathrm{mg} / \mathrm{kg}$; an intraperitoneal dose for spermiation) and ( 0.5 and $5 \mathrm{mg} / \mathrm{kg}$; intraperitoneal for ovulation, 0 and 12 hours); b) only females maintained in tanks, without males present, were induced. Eggs obtained from tanks were recollected and observed. Results. Ovulation and spermiation were verified, but embryonic development was not advanced when gametes were mixed and incubated. Testing with induced females, without males present, confirms sperm storage in ovaries, and eggs show a complete embryonic development, achieving hatching of larvae and maintenance of young fish. Conclusions. The conventional protocol using induction was effective in females and males, but the mixed products did not prove to be a viable procedure for seed production in the species. Sperm storage in females and spontaneous emission of fertilized eggs in their habitat, when they were subjected to induction, was confirmed. Form and sperm as well as physiological mechanisms occurring in fertilization are unknown.

Key words: Auchenipteridae, reproduction, siluriformes (Source: AIMS).

\section{INTRODUCCIÓN}

En general, entre las características morfológicas que se utilizan para diferenciar entre varios géneros de la familia Auchenipteridae se contemplan particularidades morfológicas, tanto en hembras como machos, que tienen funciones especializadas asociadas a los eventos reproductivos $(1,2)$. Por ejemplo, en la descripción que realizan Castillo y Brull (3) de Ageneiosus magoi se confirma que para el género es una constante la fusión de varios radios de la aleta anal, formando un órgano intromitente que en los machos permite la transferencia directa del esperma a través de un pseudopene o gonopodio. Aunque para los pimelodidos en general aparece una vesícula seminal, a la que Loir et al (4) atribuyen una función secretora, esta no se presenta en Ageneiosus; en su lugar, con posibles funciones equivalentes de secreción y almacenamiento, se desarrolla entonces una especialización del conducto deferente posterior, de forma que se incrementa su tamaño como una ampolla que finalmente desemboca en el gonopodio; esta característica, más la forma elongada del núcleo espermático y la fecundación interna, sugiere que se trata del grupo evolutivamente más avanzado en lo que se refiere al desarrollo de tracto reproductivo en machos dentro de los silúridos (4).

Castillo y Brull (3) denominan esta estructura como vesícula intermedia pero no definen su función, tanto en el proceso reproductivo como en la posible acción para la transferencia espermática en Ageneiosus. En las hembras se presenta un ensanchamiento de la porción distal de los oviductos, formándose un útero simple que aloja al pseudopene durante la cópula y que, según Sanches et al (5), en Parauchenipterus galeatus (Auchenipteridae) permite mantener el esperma viable hasta el momento de la puesta, cuando ocurre la fecundación.

Por esta serie de adaptaciones, el manejo reproductivo controlado con fines de producción se ha asumido como de difícil logro operativo y controlado para el género. En ese mismo sentido, Castillo y Koepke (6) determinan que sus observaciones en acuario sobre Ageniosus sp son las primeras que para la cópula son descritas para cualquier especie del género; consideran que puede ser un patrón de comportamiento equivalente para las diferentes especies de la familia, dada la similitud de las adaptaciones reproductivas que les son comunes.

Con base en las sinonimias ( $A$. caucanus, $A$. virgo) que para la especie referencian Maldonado et al (10), los ejemplares de doncella utilizados en este trabajo se asumen como Ageneiosus pardalis Lütken, 1874. Ya en lo que se refiere a importancia pesquera, se tiene que en el consolidado del segundo trimestre de 2011, la doncella representó el 4\% (unas 129 Ton) del volumen total desembarcado en la zona del medio y bajo Magdalena (7). Si bien se le define como especie en peligro (8), son pocos los trabajos que se enfocan hacia aspectos dirigidos a su conservación $y$, de hecho, sólo se cuenta con una referencia que aborda particularidades de su biología reproductiva (9); información complementaria sobre bioecología se encuentra reseñada en Olaya et al (9), en Maldonado et al (10) y en Mosquera et al (11). 
De trabajos experimentales con énfasis en reproducción controlada no se conoce de algún registro nacional y sobre desarrollo embrionario y larval la única referencia encontrada para Auchenipteridae (Parauchenipterus galeatus) es la de Sanches et al (5).

El objetivo del presente estudio fue describir la serie de procedimientos que finalizaron con la obtención de huevos, larvas y alevinos de doncella. Con base en las características morfológicas y la observación de particularidades reproductivas se propusieron también alternativas de manejo con metas dirigidas a disponer de una producción constante de semilla. La información se complementa con los primeros registros de seguimiento embrionario y larval que se reportan en Colombia para $A$. pardalis.

\section{MATERIALES Y MÉTODOS}

Descripción del área. El trabajo se realizó en la estación piscícola del Alto Magdalena (INCODER), localizada en el municipio de Gigante (Huila), a 960 msnm. La temperatura media es de $24^{\circ} \mathrm{C}$ y pluviosidad promedio de $2450 \mathrm{~mm}$ al año.

Ejemplares. Se dispuso de un plantel compuesto por 40 individuos, entre hembras $(454.9 \pm 128.3 \mathrm{~g})$ y machos $(155.8 \pm 31.5 \mathrm{~g})$, obtenidos en el embalse de Prado (Tolima). Desde su traslado y hasta el momento del manejo permanecieron por un período superior a 90 días en aclimatación a las condiciones de la estación. Se utilizó un estanque en tierra de $600 \mathrm{~m}^{2}$ que hace parte de la batería que en el centro se destina al mantenimiento de especies nativas, en el que, para efectos de alimentación, fueron mantenidas poblaciones de alevinos de tilapias roja, plateada y criolla.

\section{Manejo reproductivo}

Ensayo 1. En las primeras pruebas se aplicó un procedimiento convencional de inducción a la ovulación y espermiación utilizando extracto de hipófisis de carpa (EHC), en dosis preparatoria de $0.5 \mathrm{mg} \mathrm{Kg}^{-1}$ y 12 horas después una dosis definitiva de $5 \mathrm{mg} \mathrm{Kg}^{-1}$ para las hembras; en el caso de los machos se aplicó una única dosis de $4 \mathrm{mg} \mathrm{Kg}^{-1}$, simultáneamente a la dosis definitiva en las hembras. Los peces (15 hembras y 10 machos) fueron revisados continuamente $y$ la medición y registro de la temperatura se realizó cada hora, hasta el momento en que se confirmó la ovulación. Mediante extrusión los huevos fueron recolectados en recipientes secos, pesados (con aproximación a $0.1 \mathrm{~g}$ ) y se retiró una muestra para definir el diámetro pre inducción $(\mu)$ y el número de ovocitos $\mathrm{g}^{-1}$. Sobre cada una de las puestas se adicionó el esperma directamente y se procedió con la mezcla de los gametos. Después de 5 minutos se agregó agua y los huevos se mantuvieron en reposo por 30 minutos antes de ubicarlos en incubadoras experimentales de flujo vertical con $2 \mathrm{~L}$ de capacidad.

Ensayo 2. En el transcurso del ensayo anterior se detectó la presencia de huevos en el fondo de los tanques; estos fueron recolectados y al comprobar desarrollo, se programó una segunda serie de pruebas. La observación de células espermáticas en extendidos de huevos no fecundados previamente soportó también el diseño de las pruebas.

Así, en piletas y siguiendo los mismos procedimientos de inducción que fueron aplicados en la primera fase, se mantuvó un total de 3 hembras y 6 machos, los que fueron observados permanentemente con el fin de detectar la manifestación de patrones de comportamiento de cortejo y/o cópula. En dos tanques adicionales, en este mismo ensayo, se colocaron 1 y 2 hembras inducidas en piletas separadas, pero sin la presencia de machos. En todos los casos se efectuó una revisión constante sobre el fondo del tanque, con un colador plástico, con el fin de registrar la presencia y, de ser el caso, recolectar ovocitos, los que se dispusieron en recipientes de vidrio de $50 \mathrm{ml}$ y muestras del material fueron retiradas permanentemente para determinar y describir, bajo observación en microscopio y estereoscopio, avances en el desarrollo embrionario.

El conjunto de registros obtenido se presenta en forma descriptiva, con la precisión temporal de los momentos más relevantes durante las etapas de la evolución embrionaria y larval, con su correspondiente soporte fotográfico. Igualmente se describen algunos parámetros de importancia relacionados con características reproductivas y anotaciones sobre manejo en estadios tempranos.

\section{RESULTADOS}

En general, las hembras maduras son fácilmente distinguibles por el pronunciado abultamiento abdominal que presentan. La forma aserrada del primer radio de la aleta dorsal, la osificación de barbillas maxilares y la fusión de los primeros radios de la aleta anal permiten la identificación de machos maduros, en los que la emisión de esperma por masaje es también evidente. Aunque el dato debe ser precisado, la 
inducción con las dosis utilizadas se manifestó en ovulación efectiva a partir de los 625 grados $\mathrm{h}^{-1}$, en una temperatura media de $25.5^{\circ} \mathrm{C}$.

Salvo en dos ejemplares, el esperma extraído se mostró activado en todos los demás casos; con la adición de agua o suero fisiológico, las células perdieron integridad. La figura 1 muestra aglomerados de espermatozoides que parecerían estar confirmando la presencia de paquetes espermáticos en los ovarios, lo que debe ser objeto de evaluación posterior.

Los huevos de $A$. pardalis tienen una coloración que varía de amarillo a naranja, presentan doble membrana $y$, con la hidratación finalizada, el espacio perivitelino es relativamente reducido; son demersales, adherentes, y en una distribución normal de diámetro se determinó la mayor frecuencia en el rango de 1000 a 1250 $\mu$. En cantidad, se obtuvieron entre 425 y 535 huevos por gramo.

En los tanques no se observó comportamiento de cortejo y cópula entre los peces inducidos. En las pruebas que se adelantaron con hembras inducidas y sin la presencia de machos (ensayo 2), la ovulación y la presencia del material fecundado fue comprobada con la recolección de huevos viables en el fondo de las piletas. El desarrollo embrionario fue normal (Figura $2 \mathrm{a}-\mathrm{h}$ ) y la eclosión ocurrió entre las 48 y 60 horas, con una temperatura aproximada de $22^{\circ} \mathrm{C}$. Las larvas aceptan nauplios de Artemia y aparentemente no se presenta canibalismo. El seguimiento del desarrollo embrionario finalizó con la eclosión de larvas, confirmando que las hembras almacenan el esperma en los ovarios y la fecundación ocurre al parecer en el momento de la puesta.

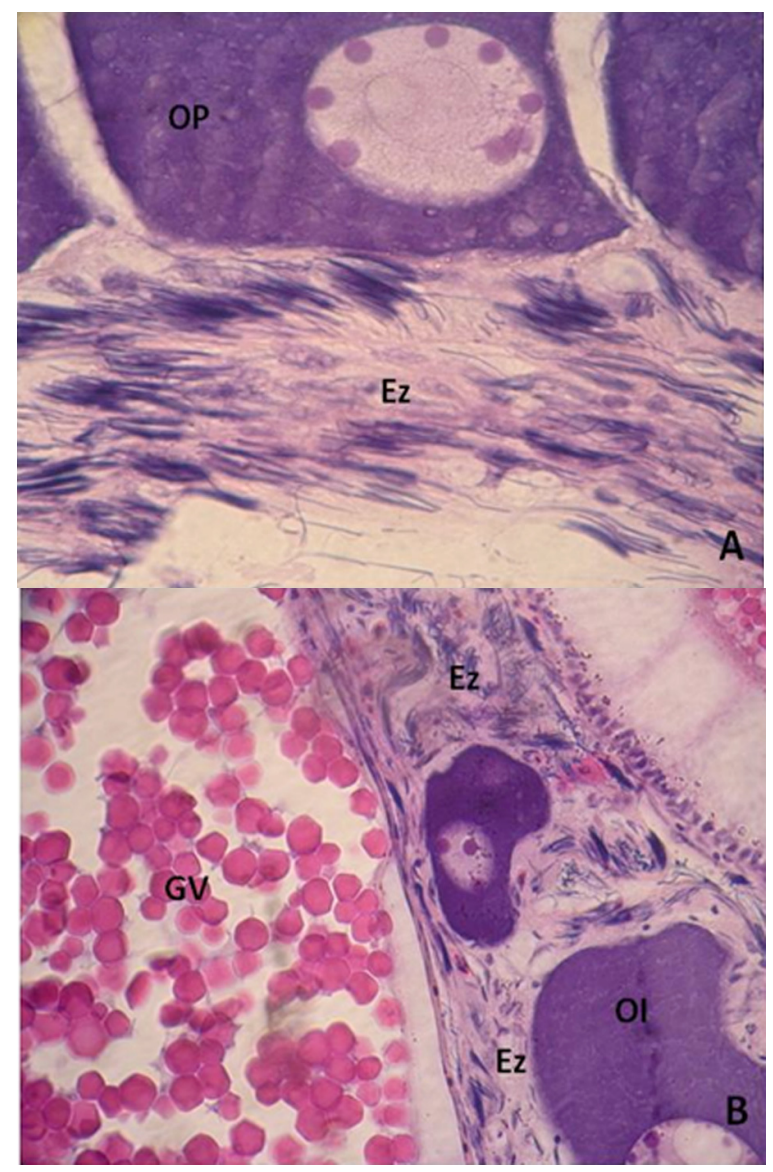

Figura 1. Cortes histológicos de ovario de doncella. A. Distribución espermática rodeando oocitos en estadio perinucleolar (OP), Ez: espermatozoides; B. Oocito maduro con granulos de vitelo (GV), oocitos inmaduros (OI) y ezpermatozoides (Ez). Técnica H E 100x.
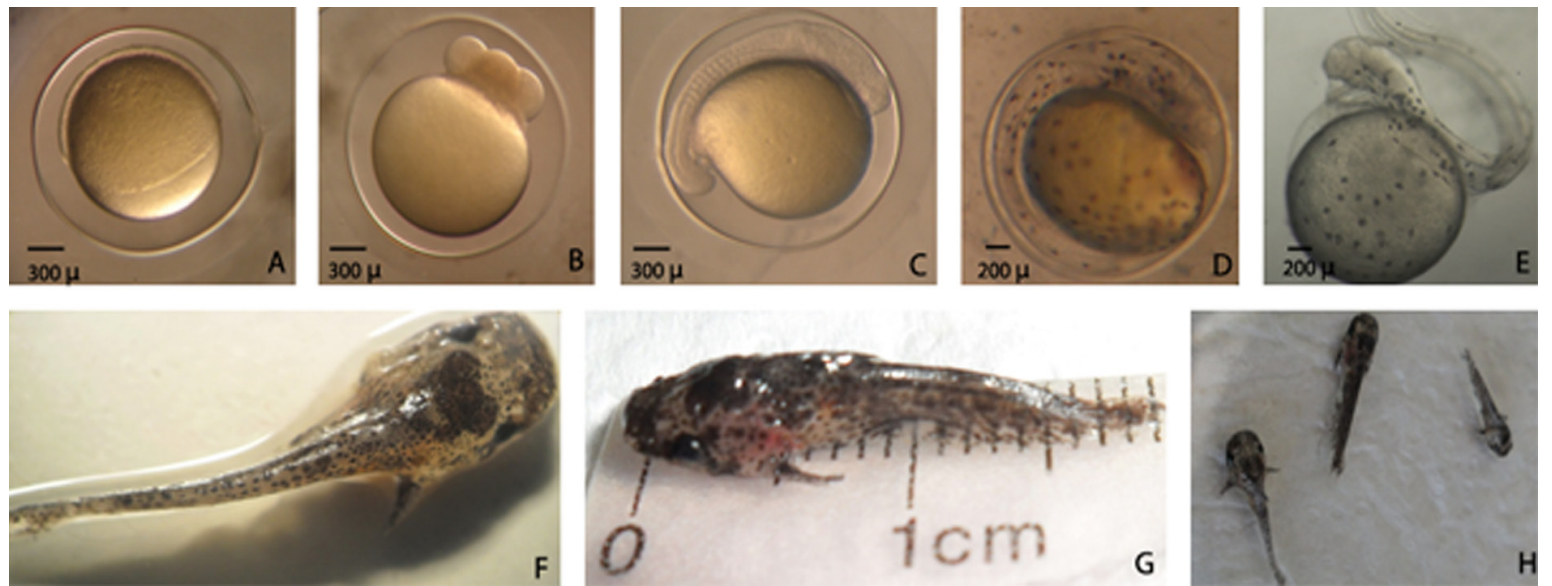

G

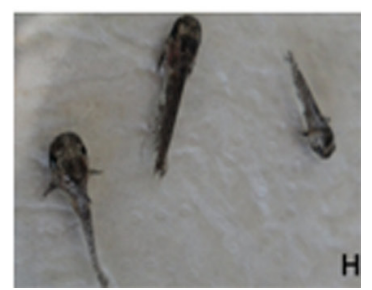

Figura 2. Desarrollo temprano en doncella (Ageneiosus pardalis). A. Gastrulación, con epibolia > al 50\%; B. Blastomeración, 4 blastómeros; C. Organogénesis inicial; D. Organogénesis tardía; E. Larva recién eclosionada; F,G,H. Alevinos. 


\section{DISCUSIÓN}

El rango de diámetro de los huevos que fue determinado (1000 a $1250 \mu$ ), coincide con el que reportan Olaya et al (9) para la especie $(1014 \pm 287 \mu)$; en comparación, esta talla es inferior a la que se registran para otras especies del género, como $A$. ucayalensis $(1.85 \mathrm{~mm})$ y $A$. valenciennesi, con $1.74 \mathrm{~mm}(5)$. En términos de manejo reproductivo, $A$. pardalis puede desovar espontáneamente mediante inducción con EHC. La puesta parece ser intermitente, en tanto se encuentran huevos de forma constante en las piletas, lo que estaría indicando una estrategia, por comprobar, que permitiría proceder con la emisión parcial en condiciones y sitios favorables. Respecto al primer ensayo se debe anotar que, puesto que existe la posibilidad de que en el momento de realizar extrusión se dispuso de huevos fértiles, las pérdidas que se presentaron en el transcurso de la incubación sugieren que para la especie deben ser valorados otros sistemas de manejo de las ovas en esta etapa.

En los machos, que en el momento de la revisión inicial presentan emisión de esperma, la aplicación de la hormona no es necesariamente un factor decisivo pues la producción seminal es relativamente abundante aún sin inducción; de cualquier forma, es evidente que valoraciones posteriores sobre las características particulares del material espermático deben ser objeto de estudio, considerando también el efecto de los inductores sobre los parámetros de calidad seminal. Con la presencia de un gonopodio se elimina la necesidad de formar paquetes espermáticos, tanto en la forma de un espermatóforo o como espermatozeugmata $(12,4)$. En los casos de fecundación interna, Burns et al (13) describen que, aún con algunas variaciones entre especies, el almacenamiento del esperma por parte de la hembra se efectúa en agrupaciones celulares diferentes, que pueden ser de alguno de los tipos mencionados. Como en este caso, en los extendidos de los huevos obtenidos por biopsia, los espermatozoides se observaron aparentemente libres, la formación por parte del macho y el almacenamiento por parte de la hembra de un empaquetamiento tipo espermatozeugmata no es aún clara.

Grier et al (12), en una precisión conceptual, diferencian entre procesos de fertilización interna y los que corresponden a una asociación interna de gametos. Establecen que en este último patrón de reproducción se incluyen especies de las que es posible obtener huevos en los que posteriormente se confirma desarrollo embrionario, sin necesidad de proceder con procedimientos de fecundación; dentro de los ovarios pueden presentar células espermáticas, pero sin embriones en formación, lo que hace la diferencia con aquellas especies en las que hay fecundación interna. Este fue precisamente el caso observado en la doncella, pues hay una evidente introducción de espermatozoides y los huevos viables obtenidos proceden de una fecundación realizada en el momento de la puesta, lo que corrobora los registros que Loir et al (4) establecen para la familia. Se desconoce para las especies de Ageneiosus el tiempo en el cual el esperma puede ser almacenado y mantenerse viable hasta la ovoposición (14), y no están descritos los mecanismos involucrados en la fecundación cuando ocurre la puesta. La recolección permanente de huevos dentro de las piletas sugiere la posibilidad de que una hembra pueda desovar en varios sitios y no de forma total en una sola localidad, lo que tendría relación con la estrategia reproductiva derivada de la asociación interna de gametos que se traduce con el almacenamiento de esperma.

La construcción de nidos o la utilización de otro tipo de estructuras para el desove son cuestiones de comportamiento reproductivo que aún deben ser definidas. También persiste el interrogante sobre la posibilidad de que se presente cópula directamente en los estanques o, que en el momento de la captura los ejemplares ya disponían de material espermático almacenado. Como se mencionó, el lote disponible se mantuvo en aclimatación por más de 90 días, por lo que la capacidad de sostener la integridad del esperma en un extenso período de tiempo es una cuestión por resolver y que aún requiere seguimiento experimental. Con base en lo anterior, se plantean las siguientes consideraciones de manejo reproductivo en cautiverio para la especie:

a) De demostrarse que existe cópula en el interior de los estanques, el control de la reproducción en cautiverio se debe puntualizar en la aplicación del inductor exclusivamente sobre las hembras, en las dosis descritas y con las cuales se confirmó respuesta positiva. En consecuencia, se puede proceder con la recolección continua de los huevos (cuya viabilidad se comprobó), directamente en los tanques en los que se mantienen las hembras inducidas.

b) La extrusión de hembras ya ovuladas es una segunda opción, lo que tendría la ventaja operativa de disponer de un sólo lote para manejo en incubación.

c) De no ocurrir cópula en cautiverio, la explicación 
que tiene la presencia de huevos fertilizados observada en estos ensayos solamente estaría dada si se asume que las hembras capturadas ya disponían del material espermático en los ovarios. Siendo esta la situación, los esquemas de obtención de larvas para un programa de producción constante pueden sustentarse en la captura de hembras maduras, proceder con la inducción descrita y disponer de huevos aplicando cualquiera de las opciones determinadas anteriormente; por tanto, se confirmaría que no hay necesidad de trabajo sobre los machos.

En definitiva y con base en lo observado, la obtención de ovocitos viables en $A$. pardalis es técnicamente factible. Ahora bien, se debe aclarar que por las características de los huevos y el tiempo relativamente largo que tiene el desarrollo embrionario que, como se mencionó, en media puede ser de unos $1250^{\circ} \mathrm{h}^{-1}$. Los aspectos relacionados con la adaptación y mejoramiento de los sistemas de incubación y el manejo de procedimientos de mantenimiento de larvas y alevinos se deben contemplar como la base de futuras líneas de investigación.

En conclusión, la respuesta a la inducción en las dosis definidas como convencionales demostró ser efectiva para promover la ovulación y la espermiación; en este caso la obtención directa por extrusión y la posterior mezcla de los gametos no se considera como un procedimiento técnicamente práctico para la producción de semilla viable para la especie.

Las estructuras anatómicas especializadas para la reproducción confirman la adaptación para la intromisión del esperma del macho hacia la hembra, lo que se corrobora con la presencia de espermatozoides en extendidos de huevos obtenidos sin haber efectuado la mezcla artificial de gametos; se desconoce tanto si se presenta cópula en estanques como el tiempo en el que el material espermático se sostiene viable en los ovarios. También queda por resolver si las células espermáticas se encuentran en forma libre dentro de los ovarios o en la forma de empaquetamiento tipo espermatozeugmata.

La ovulación y la puesta en hembras inducidas se presentan en las piletas de manejo; el material recolectado es viable en términos de desarrollo embrionario, eclosión, larvicultura y alevinaje temprano.

El tiempo de desarrollo embrionario y larval, relativamente extenso para especies de clima cálido, implica proyectar la evaluación de diferentes equipamientos para mejorar la eficiencia durante la incubación.

\section{Agradecimientos}

Los resultados obtenidos se soportan en el Convenio de Cooperación Técnica 0368 INCODER - ISAGEN - CORPORACION PROGRAMA DE DESARROLLO PARA LA PAZ DEL MAGDALENA CENTRO, entidades a las que correspondió su financiamiento. La participación y colaboración del personal técnico y operativo de la Estación, en lo referente al mantenimiento y manejo de los reproductores, además de Natalia Torres Forero, Tecnóloga en Acuicultura Continental, fueron determinantes durante las actividades de control y seguimiento sobre embriones, larvas y alevinos.

\section{REFERENCIAS}

1. Curran D. Phylogenetic relationships among the catfish genera of the family Auchenipteridae (Teleostei: Siluroidea). Copeia 1989; 2:408-419.

2. Birindelli JL. Relacoes filogenéticas da superfamília Doradoidea (Ostariophysi, Siluriformes). [Tese Doutorado]. Brasil: Instituto de Biociencias da Universidade de Sao Paulo; 2010.

3. Castillo O, Brull O. Ageneiosus magoi. Una nueva especie de Ageneiosido (Teleostei: Siluriformes), para Venezuela y algunas notas sobre su historia natural. Acta Biol Venez 1989; 12(3-4):72-87.
4. Loir M, Cauty'l C, Planquette P, Le Ba PY. Comparative study of the male reproductive tract in seven families of South-American catfishes. Aquat Living Resour 1989; 2:45-56.

5. Sanches V, Nakatani K, Bialetzki A. Morphological description of the developmental stages of Parauchenipterus galeatus (Linnaeus, 1766) (Siluriformes, Auchenipteridae) on the floodplain of the upper Paraná river. Rev Bras Biol 1999; 59(3):429-438. 
6. Castillo O, Koepke J. Aspectos sobre la reproducción del bagre Ageneiosus sp. nov. (Teleostei, Siluriformes, Ageneiosidae). En: 35 Convención Nacional de ASOVAC. Resúmenes de comunicaciones libres; Mérida (Venezuela); 1985.

7. CCI - Corporación Colombia Internacional. Sistema de Información de Pesca y Acuicultura. Boletín trimestral 60. [en línea]. 2011. [consultado Sep 12 2011]. URL Disponible en: http://www.cci.org. co/cci/cci_x/datos/BoletinesIncoder/ Mensual/2011/BolAbr-Jun2011.pdf.

8. Mojica J, Alvarez R. Ageneiosus caucanus. En: Mojica JI, Castellanos C, Usma JS, Alvarez $\mathrm{R}$, editores. Libro rojo de peces dulceacuícolas de Colombia. Bogotá: Serie libros rojos de especies amenazadas de Colombia. Instituto de Ciencias Naturales, Universidad Nacional de Colombia, Ministerio del Medio Ambiente; 2002.

9. Olaya-Nieto C, Segura-Guevara F, Brú-Cordero SB, Blanco-Viellar HM. Biología reproductiva de la Doncella Ageneiosus pardalis (Lütken, 1874 ) en el Río Sinú, Colombia. [en línea]. 2003 [consultado 20 Feb 2010]. Disponible en: www.civa2003.org.

10. Maldonado J, Vari RP, Usma JS. Checklist of the freshwater fishes of Colombia. Biota Colombiana 2008; 9(2):143-237.
11. Mosquera H, Casas J, Lozano $\mathrm{Y}$, Maturin $M$, et al. Hábitos alimenticios y factor de condición de la Doncella Ageneiosus pardalis en el río Atrato - Colombia. En: VIII Simposio Colombiano de Ictiología; Quibdó (Chocó, Colombia). Quibdó: Universidad Tecnológica del Chocó, Grupo de Zoología. 2005.

12. Grier HJ, MC Uribe, LR Parenti, De La RosaCruz G. Fecundity, the germinal epithelium, and folliculogenesis in viviparous fishes. En: Grier HJ, Uribe MC, editores. Viviparous Fishes. Florida: New Life Publications; 2005.

13. BurnsJR, MeisnerAD, WeitzmanSH, Malabarba LR, Gatten RE. Sperm and spermatozeugma ultrastructure in the inseminating catfish, Trachelyopterus lucenai (Ostariophysi: Siluriformes: Auchenipteridae). Copeia 2002; (1):173-179.

14. Legendre M, Linhart O, Billard R. Spawning and management of gametes, fertilized eggs and embryos in Siluroidei. Aquat Living Resour 1996; 9:59-80. 